\title{
Spectral parameters of electron in multi-shell open semiconductor nanotube
}

\author{
O.M. Makhanets, A.I. Kuchak, V.I. Gutsul, O.M. Voitsekhivska \\ Chernivtsi National University, \\ 2, Kotsiubynsky str., 58012 Chernivtsi, Ukraine \\ Phone: (0372)244-816,e-mail: ktf@chnu.edu.ua
}

\begin{abstract}
The spectral parameters (resonance energies and resonance widths) of electron in multi-shell open cylindrical semiconductor nanotube are theoretically investigated within the effective mass and rectangular potentials model by using the $S$-matrix approach. These parameters as functions of the nanotube thickness and axial quasimomentum are analyzed for the nanostructure composed of $\mathrm{GaAs}$ and $\mathrm{Al}_{\mathrm{x}} \mathrm{Ga}_{1-\mathrm{x}} \mathrm{As}$ semiconductors.
\end{abstract}

Keywords: nanotube, quasi-stationary state, resonance energy, resonance width.

Manuscript received 23.12.14; revised version received 12.01.15; accepted for publication 00.00.15; published online 00.00.15.

\section{Introduction}

The multi-shell semiconductor nanotubes have been recently studied both theoretically and experimentally [1-7]. The unique properties of quasi-particles (electrons, excitons and so on) in these nanostructures allow using them as basic elements for the devices of modern nanoelectronics [8-10].

The authors of ref. [3] have been grown the arrays of semiconductor nanotubes consisting of the sequence of $\mathrm{GaAs}$ and $\mathrm{Al}_{\mathrm{x}} \mathrm{Ga}_{1-\mathrm{x}} \mathrm{As}$ nanoshells by using the method of molecular beam epitaxy. This nanostructure was covered by rather thick shell of GaAs in order to avoid $\mathrm{Al}_{\mathrm{x}} \mathrm{Ga}_{1-\mathrm{x}}$ As oxidizing.

The multi-shell nanotube under study is an open one, because the potential energy of electron in GaAs is smaller than that in $\mathrm{Al}_{\mathrm{x}} \mathrm{Ga}_{1-\mathrm{x}} \mathrm{As}$. In open nanotubes, on the contrary to the closed ones, the quasi-particles can tunnel through the potential barrier into the outer medium, creating an additional channel of energy relaxation for the quasi-particles excited in the quantum well. It is clear that the quasi-particles energy spectra in these nanosystems are quasi-stationary and characterized by the resonance energies and resonance widths.

The theory of exciton and phonon stationary spectra together with the theory of electron- and exciton-phonon interaction well correlating to the experimental data and general physical considerations is already developed for the closed cylindrical and hexagonal nanotubes [5-7].

The quasi-stationary spectra of electrons, holes and excitons were theoretically studied for the sphericallysymmetric quantum dots and single cylindrical quantum wires [11-15]. In this paper, we present the theoretical study of electron quasi-stationary spectrum in multi-shell open cylindrical semiconductor nanotube. The dependences of resonance energies and resonance widths on the nanotube thickness and axial quasi-momentum of GaAs and $\mathrm{Al}_{0.4} \mathrm{Ga}_{0.6}$ As are obtained and analyzed. 


\section{Electron energy spectrum and wave functions in multi-shell cylindrical nanotube}

The multi-shell open cylindrical semiconductor nanotube consisting of inner wire with the radius $\rho_{0}$ ("0" - GaAs), barrier-shell with the thickness $\Delta_{1}$ ("1" $\mathrm{Al}_{\mathrm{x}} \mathrm{Ga}_{1-\mathrm{x}} \mathrm{As}$ ), nanotube with the thickness $h$ ("2" - GaAs) and one more barrier-shell with the thickness $\Delta_{2}$ ("3" $\mathrm{Al}_{\mathrm{x}} \mathrm{Ga}_{1-\mathrm{x}} \mathrm{As}$ ) embedded into the outer structure ("4" GaAs) is studied. The cross-section and energy scheme of this nanostructure is presented in Fig. 1. The potential energy of electron in outer medium is smaller than that in barrier-shells, thus the system is an open one, and the electron energy spectrum is quasi-stationary.

Considering the symmetry of the system, all further calculations were performed in the cylindrical coordinate system $(\rho, \varphi, z)$ with $O z$ axis directed along the axial axis of nanotube. The effective masses and potential energies of electron are fixed as

$$
\begin{gathered}
\mu(\rho)=\left\{\begin{array}{l}
\mu_{0}, \\
\mu_{1}
\end{array}\right. \\
U(\rho)=\left\{\begin{array}{lll}
0, & 0 \leq \rho \leq \rho_{0}, & \rho_{1} \leq \rho \leq \rho_{2}, \\
U_{0}, & \rho_{0}<\rho<\rho_{1}, & \rho_{2} \leq \rho \leq \rho_{3} .
\end{array}\right.
\end{gathered}
$$

The stationary Schroedinger equation

$$
\hat{H}(\rho, \varphi, z) \Psi(\rho, \varphi, z)=E \Psi(\rho, \varphi, z)
$$

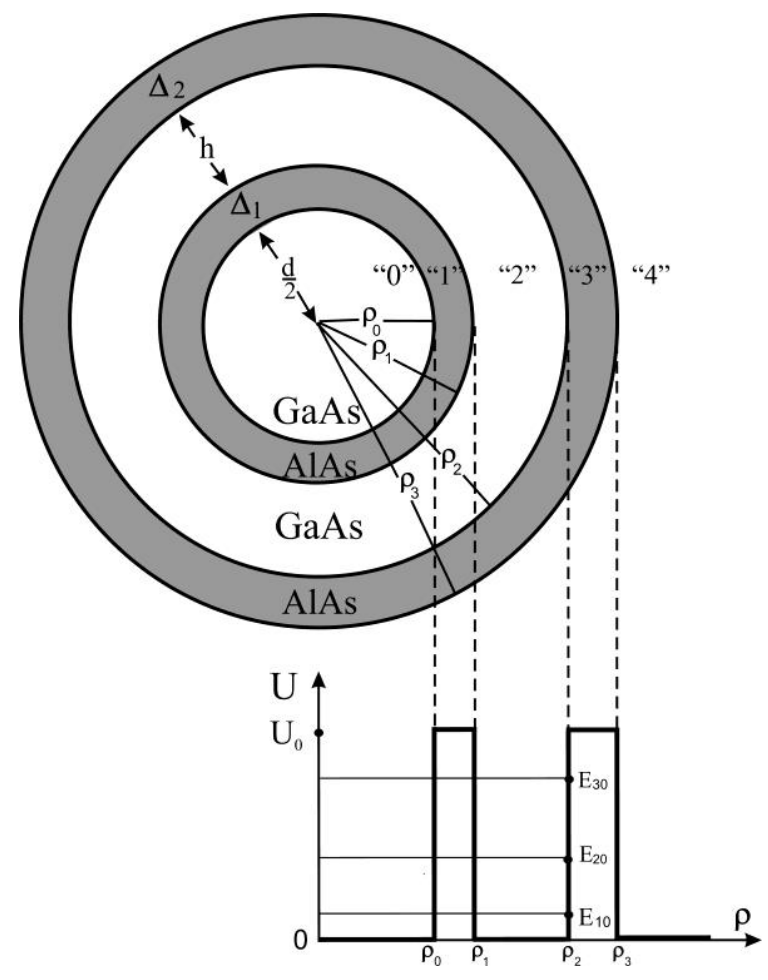

Fig. 1. Cross-section and energy scheme of multi-shell nanotube. with Hamiltonian

$$
\begin{aligned}
& \hat{H}(\rho, \varphi, z)=-\frac{\hbar^{2}}{2 \rho}\left[\frac{\partial}{\partial \rho}\left(\frac{\rho}{\mu(\rho)} \frac{\partial}{\partial \rho}\right)+\frac{1}{\mu(\rho)} \frac{1}{\rho} \frac{\partial^{2}}{\partial \varphi^{2}}\right]- \\
& -\frac{\hbar^{2}}{2 \mu(\rho)} \frac{\partial^{2}}{\partial z^{2}}+U(\rho)
\end{aligned}
$$

was solved in order to obtain the electron energy spectrum and wave functions. Considering the symmetry, the latter $(\Psi(\rho, \varphi, z))$ is written as

$\psi_{m k}(\vec{r})=\frac{1}{\sqrt{2 \pi L}} R_{m k}(\rho) e^{i k z} e^{i m \varphi}$.

Here, $k$ - the axial quasi-momentum; $m=0, \pm 1$, $\pm 2, \ldots-$ magnetic quantum number; $L-$ the effective length of electron movement along the axial axis of nanotube. Substituting (4) and (3) into the equation (2), the variables $(\rho, \varphi, z)$ are separated and the equation for the radial wave functions $R_{m k}(\rho)$ is obtained

$$
\begin{aligned}
& \left\{-\frac{\hbar^{2}}{2}\left[\frac{1}{\rho} \frac{\partial}{\partial \rho}\left(\frac{\rho}{\mu(\rho)} \frac{\partial}{\partial \rho}\right)-\frac{m^{2}}{\rho^{2} \mu(\rho)}-\frac{k^{2}}{\mu(\rho)}\right]+\right. \\
& +U(\rho)-E\} R_{m k}(\rho)=0 .
\end{aligned}
$$

This equation is exactly solved for each part of nanostructure. The solutions are written as

$$
R_{m k}(\rho)=\left\{\begin{array}{r}
R_{m k}^{(0)}(\rho)=A_{m}^{(0)}\left[H_{m}^{-}\left(k_{0} \rho\right)+H_{m}^{+}\left(k_{0} \rho\right)\right], \\
\rho<\rho_{0} \\
R_{m k}^{(1)}(\rho)=A_{m}^{(1)}\left[H_{m}^{-}\left(i k_{1} \rho\right)+S_{m k}^{(1)}(E) H_{m}^{+}\left(i k_{1} \rho\right)\right], \\
\rho_{0} \leq \rho \leq \rho_{1} \\
R_{m k}^{(2)}(\rho)=A_{m}^{(2)}\left[H_{m}^{-}\left(k_{0} \rho\right)+S_{m k}^{(2)}(E) H_{m}^{+}\left(k_{0} \rho\right)\right], \\
\rho_{1}<\rho \leq \rho_{2} \\
R_{m k}^{(3)}(\rho)=A_{m}^{(3)}\left[H_{m}^{-}\left(i k_{1} \rho\right)+S_{m k}^{(3)}(E) H_{m}^{+}\left(i k_{1} \rho\right)\right], \\
\rho_{2}<\rho \leq \rho_{3} \\
R_{m k}^{(4)}(\rho)=A_{m}^{(4)}\left[H_{m}^{-}\left(k_{0} \rho\right)+S_{m k}(E) H_{m}^{+}\left(k_{0} \rho\right)\right], \\
\rho_{3}<\rho
\end{array}\right.
$$

where

$$
k_{0}=\sqrt{\frac{2 \mu_{0}}{\hbar^{2}} E-k^{2}}, k_{1}=\sqrt{\frac{2 \mu_{1}}{\hbar^{2}}\left(U_{0}-E\right)+k^{2}},
$$

$H_{m}^{-}, H_{m}^{+}$are the Hankel functions of the whole order, $S_{m k}(E)$ is the scattering matrix.

Using the condition of wave function and its density of current continuity at all nanotube interfaces

$$
\left\{\begin{array}{l}
R_{m k}^{(p)}\left(\rho_{p}\right)=R_{m k}^{(p+1)}\left(\rho_{p}\right) \\
\left.\frac{1}{\mu_{p}} \frac{\partial R_{m k}^{(p)}(\rho)}{\partial \rho}\right|_{\rho=\rho_{p}}=\left.\frac{1}{\mu_{p+1}} \frac{\partial R_{m k}^{(p+1)}(\rho)}{\partial \rho}\right|_{\rho=\rho_{p}} \quad(p=0,1,2,3)
\end{array}\right.
$$

(C) 2015, V. Lashkaryov Institute of Semiconductor Physics, National Academy of Sciences of Ukraine 
together with the normality condition

$$
\int_{0}^{\infty} R_{k_{0} m k}^{*}(\rho) R_{k_{0}^{\prime} m k}(\rho) \rho d \rho=\delta\left(k_{0}-k_{0}^{\prime}\right),
$$

we obtained all unknown coefficients $\left(A_{m}^{(i)}, S_{m k}^{(i)}\right)$ and analytical expression for the $S$-matrix

$$
\begin{aligned}
& S_{m k}= \\
& =\frac{\frac{i k_{1} \mu_{0}}{k_{0} \mu_{1}} \Phi_{m k}^{(3)^{\prime}}\left(i k_{1} \rho_{3}\right) H_{m}^{-}\left(k_{0} \rho_{3}\right)-\Phi_{m k}^{(3)}\left(i k_{1} \rho_{3}\right) H_{m}^{-^{\prime}}\left(k_{0} \rho_{3}\right)}{\Phi_{m k}^{(3)}\left(i k_{1} \rho_{3}\right) H_{m}^{+^{\prime}}\left(k_{0} \rho_{3}\right)-\frac{i k_{1} \mu_{0}}{\mu_{1} k_{0}} \Phi_{m k}^{(3)^{\prime}}\left(i k_{1} \rho_{3}\right) H_{m}^{+}\left(k_{0} \rho_{3}\right)},
\end{aligned}
$$

where

$$
\Phi_{m k}^{(3)}\left(i k_{1} \rho_{3}\right)=H_{m}^{-}\left(i k_{1} \rho_{3}\right)+S_{m k}^{(3)} H_{m}^{+}\left(i k_{1} \rho_{3}\right),
$$

$S_{m k}^{(3)}=$

$$
=\frac{\frac{k_{0} \mu_{1}}{i k_{1} \mu_{0}} \Phi_{m k}^{(2)^{\prime}}\left(k_{0} \rho_{2}\right) H_{m}^{-}\left(i k_{1} \rho_{2}\right)-\Phi_{m k}^{(2)}\left(k_{0} \rho_{2}\right) H_{m}^{-\prime}\left(i k_{1} \rho_{2}\right)}{\Phi_{m k}^{(2)}\left(k_{0} \rho_{2}\right) H_{m}^{+^{\prime}}\left(i k_{1} \rho_{2}\right)-\frac{k_{0} \mu_{1}}{i k_{1} \mu_{0}} \Phi_{m k}^{(2)^{\prime \prime}}\left(i k_{1} \rho_{3}\right) H_{m}^{+}\left(i k_{1} \rho_{2}\right)},
$$$$
\Phi_{m k}^{(2)}\left(k_{0} \rho_{2}\right)=H_{m}^{-}\left(k_{0} \rho_{2}\right)+S_{m k}^{(2)} H_{m}^{+}\left(k_{0} \rho_{2}\right),
$$$$
S_{m k}^{(2)}=
$$

$$
=\frac{\frac{i k_{1} \mu_{0}}{\mu_{1} k_{0}} \Phi_{m k}^{(1)^{\prime}}\left(i k_{1} \rho_{1}\right) H_{m}^{-}\left(k_{0} \rho_{1}\right)-\Phi_{m k}^{(1)}\left(i k_{1} \rho_{1}\right) H_{m}^{-^{\prime}}\left(k_{0} \rho_{1}\right)}{\Phi_{m k}^{(1)}\left(i k_{1} \rho_{1}\right) H_{m}^{+^{\prime}}\left(k_{0} \rho_{1}\right)-\frac{i k_{1} \mu_{0}}{k_{0} \mu_{1}} \Phi_{m k}^{(1)^{\prime}}\left(i k_{1} \rho_{1}\right) H_{m}^{+}\left(k_{0} \rho_{1}\right)},
$$

$\Phi_{m k}^{(1)}\left(i k_{1} \rho_{1}\right)=H_{m}^{-}\left(i k_{1} \rho_{1}\right)+S_{m k}^{(1)} H_{m}^{+}\left(i k_{1} \rho_{1}\right)$,

$$
S_{m k}^{(1)}=\frac{\frac{k_{0} \mu_{1}}{i k_{1} \mu_{0}} J_{m}^{\prime}\left(k_{0} \rho_{0}\right) H_{m}^{-}\left(i k_{1} \rho_{0}\right)-J_{m}\left(k_{0} \rho_{0}\right) H_{m}^{-}\left(i k_{1} \rho_{0}\right)}{J_{m}\left(k_{0} \rho_{0}\right) H_{m}^{+^{\prime}}\left(i k_{1} \rho_{0}\right)-\frac{k_{0} \mu_{1}}{i k_{1} \mu_{0}} J_{m}^{\prime}\left(k_{0} \rho_{0}\right) H_{m}^{+}\left(i k_{1} \rho_{0}\right)} .
$$

According to the general theory, the poles of $S$ matrix in the complex energy plane define the electron resonance energies $E_{n_{\mathrm{\rho}} m}(k)$ and resonance widths $\Gamma_{n_{\rho} m}(k)$ or its life times $\tau_{n_{\rho} m}(k)=\hbar / \Gamma_{n_{\rho} m}(k)$ in quasistationary states

$$
\tilde{E}_{n_{\rho} m}(k)=E_{n_{\rho} m}(k)-i \Gamma_{n_{\rho} m}(k) / 2 .
$$

The quantum number $n_{\rho}$ denotes $S$-matrix poles at fixed $m$.

Finally, the formulas (10)-(12) definitely determine the spectral parameters (resonance energies and widths) of electron in multi-shell open cylindrical semiconductor nanotube.

\section{Analysis of results}

The numeric calculation of electron spectral parameters was performed for the multi-shell nanotube composed of $\mathrm{GaAs} / \mathrm{Al}_{\mathrm{x}} \mathrm{Ga}_{1-\mathrm{x}} \mathrm{As}$ semiconductors with the parameters: $\mu_{0}=0.063 m_{0}, \mu_{1}=(0.063+0.083 x) m_{0}\left(m_{0}\right.$ is the mass of free electron in vacuum), $U_{0}=0.57\left(1.155 x+0.37 x^{2}\right)$ and the lattice parameter $a_{\mathrm{GaAs}}=5.65 \AA$.

In Fig. 2, the resonance energies $E_{n_{\mathrm{p}} m}(a)$ and widths $\Gamma_{n_{\mathrm{p}} m}(b)$ are presented as functions of the nanotube thickness $h$ at $k=0, m=0$, a fixed radius of the inner wire $\rho_{0}=10 a_{\mathrm{GaAs}}$ and barrier-shells thicknesses $\Delta_{1}=$ $\Delta_{2}=4 a_{\text {GaAs. }}$. Figure proves that both the resonance energies and widths non-monotonously depend on the nanotube thickness. Herein, the resonance energies as functions of $h$ are the sequence of horizontal and decaying plots, while at the functions of resonance widths the clearly visible maxima are observed for small $\Gamma$. Horizontal plots in Fig. 2 a correspond to the states where electron is located in the inner wire with a higher probability. In the states corresponding to the decaying plots, electron is mainly located in nanotube. Its increasing thickness causes the decrease in the resonance energy.

The dependences of resonance widths on $h$ (Fig. 2b) are explained in the following way. Let us observe for example the ground electron state $\left(n_{\rho}=1\right.$, $m=0)$ : at $h=0$ the nanotube is absent and electron is localized in the inner wire, in order to transit into the outer medium it has to tunnel through the rather strong potential barrier with the thickness $\Delta_{1}+\Delta_{2}$. Thus, the resonance width $\Gamma_{10}$ of energy level is small. Electron penetrates into nanotube more and more when $h$ increases. Now, it has to tunnel through the only one barrier-shell with the thickness $\Delta_{2}$ to transit into the outer medium. Consequently, the resonance width of energy level increases, approaching its maximum. Further, it only decays because the resonance energy becomes smaller and the height of the potential barrier effectively increases.

The function $\Gamma_{20}$ does not look the same as $\Gamma_{10}$ (Fig. 2b). One can see that for small $h$, the electron energy of the second quasi-stationary state rapidly decreases when $h$ increases (Fig. 2a). Electron is localized in nanotube, and its resonance width is rather large but rapidly decays due to the bigger effective height of potential barrier (Fig. 2b). It approaches the minimal magnitude at the nanotube thickness changing from $h \approx 15 a_{\mathrm{GaAs}}$ up to $h \approx 25 a_{\mathrm{GaAs}}$, when electron is located in the inner wire and its energy almost does not depend on $h$ (Fig. 2a). The electron energy $E_{20}$ decreases when $h$ increases further. The quasi-particle is localized in nanotube and function $\Gamma_{20}$ is similar to $\Gamma_{10}$ : at first it increases and then decreases only. 


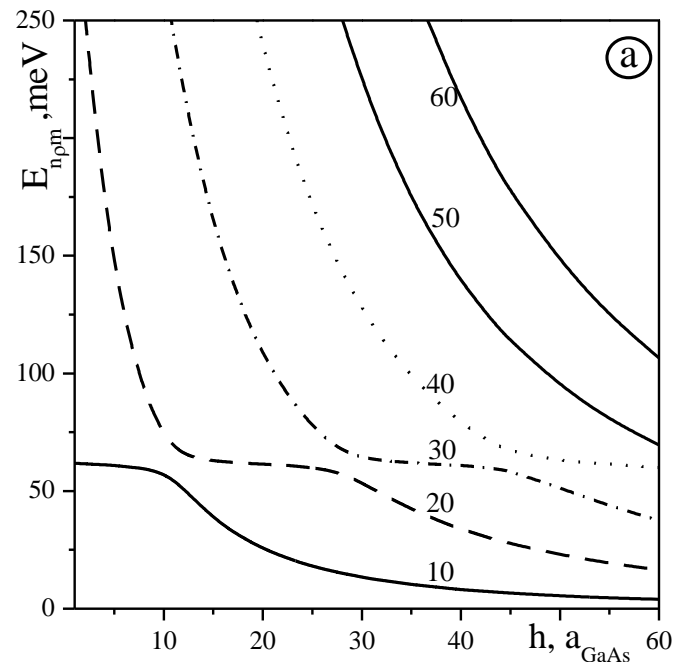

Fig. 2. Resonance energies $E_{n_{p} m}(a)$ and resonance widths $\Gamma_{n_{\mathrm{p}}}$

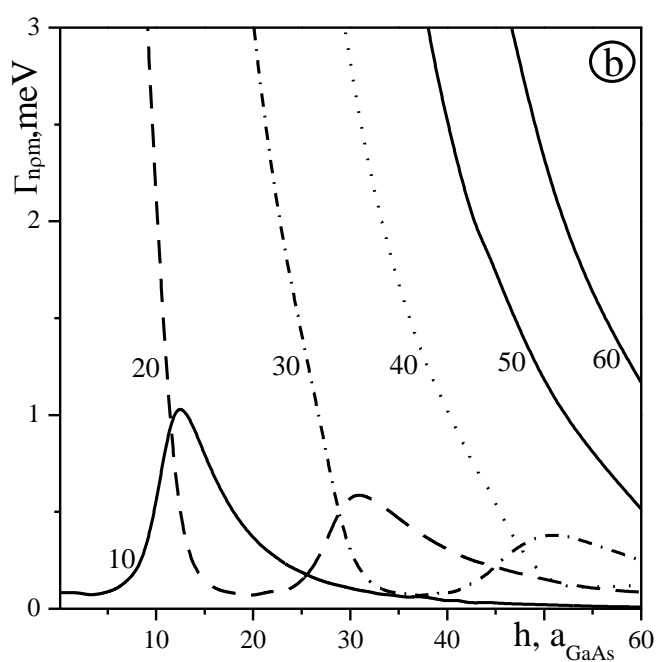

(b) as functions of the nanotube thickness $h$ at $k=0, m=0$,

fixed radius of the inner wire $\rho_{0}=10 a_{\mathrm{GaAs}}$ and barrier-shells thicknesses $\Delta_{1}=\Delta_{2}=4 a_{\mathrm{GaAs}}$.

The non-monotonous behavior of resonance widths of other energy states is also explained by different location of electron in the space of multi-shell nanostructure.

It should be noted that, contrary to the single open quantum wires where the higher energy level (over $n_{\rho}$ quantum number) at fixed $m$ has a wider resonance width [13], in the case of the studied nanotube one can see that, for example, when the nanotube thickness varies from $h \approx 12 a_{\mathrm{GaAs}}$ up to $h \approx 26 a_{\mathrm{GaAs}}, E_{20}>E_{10}$, however $\Gamma_{20}<\Gamma_{10}$. This peculiarity of spectral parameters gives opportunity to produce multi-shell nanotubes with the inverse occupied levels, which can be used as active elements of semiconductor lasers.

In Fig. 3, the electron resonance energies (Figs. 3a and 3c) and widths (Figs. 3b and 3d) are presented as functions of the axial quasi-momentum $k$ at $m=0$, fixed radius of the inner wire $\rho_{0}=10 a_{\mathrm{GaAs}}$, thicknesses of barrier-shells $\Delta_{1}=\Delta_{2}=4 a_{\mathrm{GaAs}}$, nanotube thickness $h=$ $25 a_{\mathrm{GaAs}}$ and two different concentrations $x=0.2$ and $x=0.4$. In Figs. $3 \mathrm{a}$ and $3 \mathrm{c}$, one can also see the dispersion laws of electron energy in bulk semiconductor crystals $\mathrm{GaAs}$ and $\mathrm{Al}_{\mathrm{x}} \mathrm{Ga}_{1-\mathrm{x}} \mathrm{As}$, being the composition materials of studied nanotube,

$$
E_{\mathrm{GaAs}}=\frac{\hbar^{2} k^{2}}{2 \mu_{0}}, \quad E_{\mathrm{Al}_{\mathrm{x}} \mathrm{Ga}_{1-\mathrm{x}} \mathrm{As}}=U_{0}+\frac{\hbar^{2} k^{2}}{2 \mu_{1}} .
$$

Figs. $3 \mathrm{a}$ and $3 \mathrm{c}$ show that these curves separate the plane $(E, k)$ into three regions: $\mathrm{I}$ - the region where the $S$-matrix has not any poles and, respectively, there are not any states here; II - the region where the $S$-matrix has several poles in complex energy plane defining the resonance energy and width as functions of the quasimomentum $k$; III - the region with $E>E_{\mathrm{Al}_{\mathrm{x}} \mathrm{Ga}_{1-\mathrm{x}} \mathrm{As}}(k)$ where electron is in stationary states of continuum energy spectrum.
Dependences of resonance energies in all the states (Figs. 3a and 3c, region II) are well approximated by the law

$E_{n_{\rho} m}(k)=E_{n_{\rho} m}(0)+\frac{\hbar^{2} k^{2}}{2 \mu_{n_{\rho} m}}$

and electron effective masses $\frac{1}{\mu_{n_{\mathrm{\rho}} m}}=\left.\frac{1}{\hbar^{2}} \frac{\partial^{2} E_{n_{\mathrm{\rho}} m}(k)}{\partial k^{2}}\right|_{k=0}$, as it is clear from Table, are close to its effective mass in bulk GaAs. For the higher $n_{\rho}$, the effective mass weakly increases due to the higher energy of electron, more and more tunneling into the barrier-shell where the effective mass is higher.

All the bands of resonance energies are characterized by the maximal longitudinal quasimomentum $\bar{k}_{n_{\rho} m}$ defined from the expression

$E_{n_{\rho} m}(k)=U_{0}+\frac{\hbar^{2}\left(\bar{k}_{n_{\mathrm{\rho}} m}\right)^{2}}{2 \mu_{1}}$.

The higher magnitudes of maximal quasimomentum correspond to the lower energy levels. Besides, $\bar{k}_{n_{\mathrm{\rho}} m}$ increases for the higher concentrations $x$ (Figs. 3a and 3b).

Table. Effective mass of electron moving along the nanotube axis.

\begin{tabular}{|c|c|c|c|c|c|c|c|}
\hline$x$ & \multicolumn{3}{|c|}{0.2} & \multicolumn{3}{c|}{0.4} \\
\hline$n_{\rho} m$ & 10 & 20 & 30 & 10 & 20 & 30 & 40 \\
\hline$\mu_{n_{\rho} m}$ & 0.0642 & 0.0649 & 0.0656 & 0.0638 & 0.0646 & 0.052 & 0.067 \\
\hline
\end{tabular}



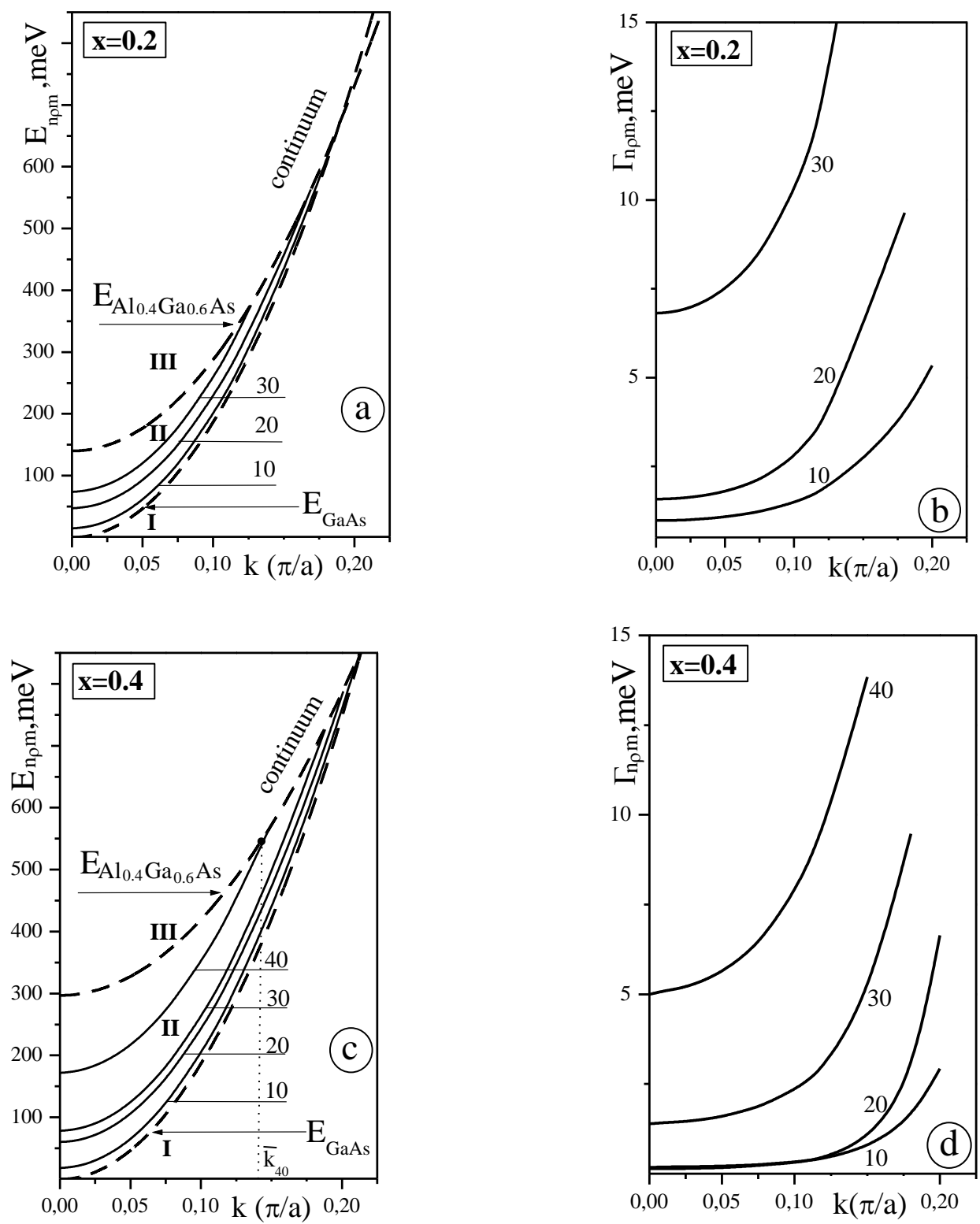

Fig. 3. Electron resonance energies $(a, c)$ and resonance widths $(b, d)$ as functions of axial quasi-momentum $k$ at $m=0$, fixed radius of the inner wire $\rho_{0}=10 a_{\mathrm{GaAs}}$, thicknesses of barrier-shells $\Delta_{1}=\Delta_{2}=4 a_{\mathrm{GaAs}}$, nanotube thickness $h=25 a_{\mathrm{GaAs}}$ and concentrations $x=0.2$ and $x=0.4$.

Figs. $3 \mathrm{~b}$ and $3 \mathrm{~d}$ prove that the resonance widths of all the quasi-stationary states rapidly increase for the higher longitudinal quasi-momentum. It is clear, because the increasing quasi-momentum causes the decrease of the effective height of potential barrier for the electron tunneling into the out of the barrier space with a higher probability. This feature of nanotubes can be used in devices - separators of electrons over their velocities, eliminating the rapid ones through the barrier and leaving the slow ones.

\section{Conclusions}

The spectral parameters of electron in multi-shell open cylindrical semiconductor nanotube were investigated within the effective mass and rectangular potentials model by using the $S$-matrix approach. These parameters as functions of the nanotube thickness and axial quasi-momentum have been analyzed for the nanostructure composed of $\mathrm{GaAs}$ and $\mathrm{Al}_{\mathrm{x}} \mathrm{Ga}_{1-\mathrm{x}} \mathrm{As}$ semiconductors.

It has been shown that both the resonance energies and widths non-monotonously depend on the nanotube thickness. For the resonance energies, the nonmonotonous character is pronounced as alternating horizontal and decaying plots, while the functions of resonance widths display clearly observed maxima and minima. This behavior of spectral parameters is caused by the complicated character of probability density of electron location in the space of nanostructure. 
It has been ascertained that all minibands of resonance energies are characterized by the effective masses that are close to the electron effective mass in bulk GaAs and by the maximal longitudinal quasimomentum. The electron resonance energies and width monotonously increase when the axial quasi-momentum increases.

\section{References}

1. P. Mohan, J. Motohisa, T. Fukui, Realization of conductive InAs nanotubes based on latticemismatched InP/InAs core-shell nanowires // Appl. Phys. Lett. 88(1), p. 013110-013115 (2006).

2. P. Mohan, J. Motohisa, T. Fukui, Fabrication of InP/InAs/InP core-multishell heterostructure nanowires by selective area metalorganic vapour phase epitaxy // Appl. Phys. Lett. 88(13), p. 133105133118 (2006).

3. M. Heigoldt, J. Arbiol, D. Spirkoska et al., Long range epitaxial growth of prismatic heterostructures on the facets of catalyst-free GaAs nanowires // J. Mater. Chem. 19, p. 840-848 (2009).

4. A. Fontcuberta i Morral, D. Spirkoska, J. Arbiol, M. Heigoldt, J. R. Morante, G. Abstreiter, Prismatic quantum heterostructures synthesized on molecular-beam epitaxy GaAs nanowires // Small, 4, p. 899-903 (2008)

5. O.M. Makhanets, N.R. Tsiupak, O.M. Voitsekhivska, Intensities of quantum transitions in hexagonal nanotubes within the exciton spectral range // Semiconductor Physics, Quantum Electronics \& Optoelectronics, 15(2), p. 156-161 (2012).

6. O.M. Makhanets, N.R. Tsiupak, V.I. Gutsul, Phonon spectra and electron-phonon interaction in a combined cylindrical semiconductor nanotube // Ukr. J. Phys. 57(10), p. 1060-1068 (2012).
7. O.M. Makhanets, V.I. Gutsul, N.R. Tsiupak, O.M. Voitsekhivska, Exciton spectrum in multi-shell hexagonal semiconductor nanotube // Condensed Matter Physics.15(3), p. 33704: 1-9 (2012).

8. J. Arbiol, E. Comini, G. Faglia, G. Sberveglieri and J.R. Morante, Orthorhombic $\mathrm{Pbcn} \mathrm{SnO}_{2}$ nanowires for gas sensing applications // J. Cryst. Growth, 310(1), p. 253-260 (2008).

9. Y. Qin, X.D. Wang and Z.L. Wang, Microfibrenanowire hybrid structure for energy scavenging // Nature, 451(7180), p. 809-813 (2008).

10. F. Qian, Y. Li, S. Gradeak, H.-G. Park, Y. Dong, Y. Ding, Z.L. Wang and C.M. Lieber, Multiquantum-well nanowire heterostructures for wavelength-controlled lasers // Nature Materials, 7(9), p. 701-706 (2008)

11. M. Tkach and V. Holovatsky, Lifetime of electron and hole quasi-stationary states in a spherical nanoheterosystem b-HgS/ b-CdS/ b-HgS // Ukr. J. Phys., 45(8), p. 972-975 (2000).

12. N.V. Tkach, V.A. Holovatsky, O.N. Voitsekhivska, On the spectra of electrons and holes in an open spherical nanoheterostructure (through the example of $\left.\mathrm{GaAs} / \mathrm{Al}_{\mathrm{x}} \mathrm{Ga}_{1-\mathrm{x}} \mathrm{As} / \mathrm{GaAs}\right)$ // Semiconductors, 34(5), p. 583-588 (2000).

13. N.V. Tkach and V.A. Golovatskii, Quasi-stationary states of electrons and holes in an open composite cylindrical quantum wire // Physics of the Solid State, 43(2), p. 365-372 (2001).

14. N.V. Tkach, Yu.A. Seti, Evolution of the electron spectrum of spherically symmetric states under the transition from a closed double-well dot to a simple open spherical quantum one // Ukr. J. Phys. 51(9), p. 908-916 (2006).

15. N.V. Tkach, Yu.A. Seti, G.G. Zegrya, Electron properties of open semiconductor quantum dots // Technical Phys. Lett. 33(1), p. 35-39 (2007). 